\title{
Impacto do desequilibrio estático e dinâmico no risco de quedas em indivíduos com ataxia espinocerebelar
}

Impact of static and dynamic imbalance in the risk of falls in paients with spinocerebellar ataxia

\section{Marisa Maia Leonardi', Gabriela de Jesus Lopes ${ }^{1}$, Poliana Penasso Bezerra ${ }^{2}$, Ana Paula Oliveira Borges ${ }^{3}$}

\section{RESUMO}

Introdução. A ataxia espinocerebelar é uma patologia hereditária com alterações neurodegenerativas caracterizada pela progressiva ataxia, oscilação postural e reações de equilíbrio atrasadas. Esses indivíduos apresentam grande propensão a quedas. A avaliação do equilíbrio é fundamental para prevenir quedas nessa população. Objetivo. Avaliar o equilíbrio estático e dinâmico em indivíduos com ataxia espinocerebelar, a fim de correlacionar com a propensão ao risco de quedas. Método. Foram avaliados sete pacientes com diagnóstico clínico de ataxia espinocerebelar. Para avaliação do equilíbrio foi utilizada a escala de equilíbrio de Berg e para averiguação do risco de quedas foi utilizada a escala de equilíbrio especifico a atividade. Para análise estatística foi realizado o teste de correlação de Pearson. Resultados. Evidenciou-se uma correlação positiva (r: $0,86)$ entre o déficit de equilíbrio e o risco de quedas nos indivíduos avaliados. Conclusão. Os dados do presente estudo evidenciaram que a marcha atáxica e déficits de coordenação proporcionam um desequilíbrio estático e dinâmico nesses indivíduos levando a maior propensão a quedas freqüentes.

Unitermos. Cerebelo, Ataxia espinocerebelares, Equilíbrio Musculosquelético, Acidentes por Quedas.

Citação. Leonardi MM, Lopes GJ, Bezerra PP, Borges APO. Impacto do desequilibrio estático e dinâmico no risco de quedas em indivíduos com ataxia espinocerebelar.

\section{SUMMARY}

Introduction. The spinocerebellar ataxia is a hereditary pathology with neurodegenerative alterations characterized by gradual ataxia, postural oscillation, and delayed balance reactions. These individuals present great propensity to fall. The balance evaluation is important to prevent falls in this population. Objective. The aim of the study was to evaluate the balance in individuals with spinocerebellar ataxia, in order to correlate it with the risk to fall. Method. Balance and risks of fall was evaluated in seven patients with clinical diagnosis of spinocerebellar ataxia, with Berg scale. The statistical analysis was Pearson's Correlation. Results. There was a positive correlation ( $\mathrm{r}: 0.86)$ between the balance deficit and the risk of falls. Conclusion. Our data suggest that the ataxic gait and coordination deficits lead to a static and dynamic imbalance and propensity to fall.

Keywords. Cerebellum, Spinocerebellar Ataxia, Musculoskeletal Equilibrium, Accidental Falls

Citation. Leonardi MM, Lopes GJ, Bezerra PP, Borges APO. Impact of static and dynamic imbalance in the risk of falls in patients with spinocerebellar ataxia.
Trabalho realizado no Departamento de Neurologia Clínica e Fisioterapia Neuro-Funcional da Universidade de Franca, Franca-SP, Brasil.Franca, Franca-SP, Brasil.

1. Graduanda do Curso de Fisioterapia da Universidade de Franca UNIFRAN, Franca-SP, Brasil.

2. Fisioterapeuta, Mestre, Professora de Fisioterapia da UNIFRAN, Franca-SP, Brasil.

3. Fisioterapeuta, Mestre, Professora de Fisioterapia da UNIFRAN e UNIARAXÁ, Franca-SP, Brasil.
Endereço para correspondência: Ana Paula O Borges Clínica de Fisioterapia - UNIFRAN Av. Armando S Oliveira, 201 CEP 14.404-600, Caixa Postal 082, Franca-SP, Brasil e-mail: anaproliveira@unifran.br 


\section{INTRODUÇÃO}

O cerebelo apresenta grande importância para o Sistema Nervoso Central (SNC) pelas suas funções e inter-relações que desempenha ${ }^{1}$. As principais funções do cerebelo envolvem coordenação da atividade motora, equilíbrio e tônus muscular. Além disso, o cerebelo é vital no controle das atividades musculares rápidas, correção e aprendizado motor. Doenças e distúrbios cerebelares produzem deficiências na velocidade, amplitude e força do movimento. Dessa forma, a perda dessa área do encéfalo pode resultar em incoordenação dessas atividades motoras ${ }^{2}$.

Dentre as disfunções cerebelares, a ataxia espinocerebelar é uma patologia hereditária de alterações neurodegenerativas, caracterizada clinicamente por progressiva oscilação postural associada com disartria, disfagia e sinais piramidais e extrapiramidais3. Geneticamente, essa desordem pode ser dividida em: autossômica dominante, autossômica recessiva e casos isolados ${ }^{3,4}$.

Com os avanços recentes na área das ataxias espinocerebelares poder-se-ia utilizar uma subclassificação destas entidades em três grupos distintos, baseados no principal mecanismo patogênico. $O$ primeiro grupo seria de doenças da poliglutamina (como as ataxias espinocerebelares tipos 1, 2, 3, 7, 17), as quais resultam da ação de proteínas (ataxinas) com tratos de poliglutamina, que são tóxicos. O segundo grupo seria representado pelas canalopatias (incluindo as ataxias espinocerebelares tipos $6 \mathrm{e}$ episódicas tipo 1, 2 por exemplo), as quais resultam da disfunção de canais de potássio e de cálcio. $\mathrm{O}$ último grupo seria representado pelos transtornos de expressão de genes (ataxias espinocerebelares tipos 8,10 e 12), que resultam de expansões repetidas localizadas fora das regiões de codificação, mas que podem alterar a expressão gênica. As ataxias espinocerebelares tipos 4, 5, 9, 11, 13-16, 18,19, 21, 23, 24 e 25 são ainda consideradas de etiologia idiopática ${ }^{5}$.

As disfunções no cerebelo resultam em três déficits principais: hipotonia, ataxia e tremor intencional. O comprometimento da marcha pode ser resultado de erros na velocidade e nível absoluto de força de contração muscular e podem acompanhar a dismetria de movimentos isolados. Além disso, os músculos da fonação são acometidos levando a disartria $^{6,7}$.

O equilíbrio pode ser definido como o processo pelo qual o SNC gera os padrões de atividade muscular necessários para regular a relação entre centro de massa e base de sustentação. Essa atividade é um processo complexo que envolve os esforços coordenados de mecanismos aferentes (visual, vestibular e proprioceptivo) e mecanismos eferentes (força muscular e flexibilidade articular) ${ }^{8,9}$.

A conservação da estabilidade é um processo dinâmico, que envolve o estabelecimento de equilíbrio entre as forças de estabilização e desestabilização. Essas respostas de equilíbrio são dependentes da interação entre o individuo, a tarefa e o ambiente, e são desencadeadas pela precisão dos impulsos sensoriais específicos e integridade dos processos motores ${ }^{10}$.

Uma imensa variedade de problemas pode contribuir para o descontrole postural do paciente com degeneração cerebelar. Os problemas de coordenação que se manifestam nas estratégias de movimento postural incluem: alterações de formação de seqüência, problemas de ativação oportuna das sinergias de resposta muscular, distúrbios associados à classificação da atividade muscular postural e problemas em adaptar as respostas motoras às condições mutáveis da tarefa ${ }^{11}$.

A marcha atáxica e déficits de coordenação proporcionam um desequilíbrio estático e dinâmico nesses indivíduos levando às quedas freqüentes ${ }^{12,13}$. A queda ou a perda do equilíbrio pode ser definida pelo movimento do centro da massa para fora dos limites da base de apoio. Clinicamente, uma queda pode ser definida como um contato não planejado e inesperado com a superfície de apoio (como o solo, uma cadeira, uma parede ou qualquer outro obstáculo) ${ }^{14}$. Aqueles que sofrem quedas apresentam um grande declínio funcional nas atividades de vida diária e nas atividades sociais, com aumento do risco de institucionalização ${ }^{15}$.

Podemos verificar que a postura e o equilíbrio envolvem tanto a capacidade de se recuperar da instabilidade como a habilidade de antecipar e mover-se de forma que o ajudem a evitar a instabilidade. Lesões no cerebelo podem resultar em oscilação postural e reações de equilíbrio atrasadas. $\mathrm{O}$ uso da visão pode não ser eficaz na prevenção da perda do equilíbrio. Esses indivíduos apresentam, dessa forma, grande propensão a quedas. Assim, a avaliação do equilíbrio é fundamental para ajudar a prevenir as quedas nessa população de indivíduos. Assim, o presente estudo teve como objetivo averiguar a relação entre o equilíbrio estático e dinâmico em indivíduos com ataxia espinocerebelar e a propensão a quedas. 


\section{MÉTODO}

\section{Amostra}

Foram avaliados 7 pacientes tendo como diagnóstico clínico a ataxia espinocerebelar, sendo 1 homem e 6 mulheres, com idade média de 38 $\pm 9,6$ anos. Todos apresentavam sinais clínicos de lesão há pelo menos 4 anos. Este estudo foi realizado no setor de neurologia da clínica-escola de fisioterapia da UNIFRAN. Ele foi previamente aprovado pelo Comitê de Ética em Pesquisa (protocolo $n^{\circ}$ 085/05) e os pacientes foram informados sobre os procedimentos e assinaram um termo de consentimento livre e esclarecido.

\section{Procedimentos}

Para a avaliação do equilíbrio foi utilizada a escala de equilíbrio de Berg16, a qual são utilizadas 14 tarefas, em que o número 4 é usado para indicar que o paciente realiza a tarefa de forma independente e de acordo com os critérios de tempo e distância, e o número 0 é usado para indicar incapacidade de realizar a tarefa; nesta avaliação é possível um máximo de 56 pontos $^{16}$. Para averiguação do risco de quedas, foi utilizada a escala de Equilíbrio Especifico à Atividade (EEEA) ${ }^{17}$, que é um questionário de 16 itens correspondentes a confiança adquirida pelo paciente na execução de 16 atividades dentro da casa, classificada com uma pontuação de 0 (não há confiança) a 100 (100\% de confiança). O total das classificações foram somados e divididos por 16 , para obter a pontuação para cada questão da escala ${ }^{17}$. As pontuações correspondentes a cada escala, descrito pelas avaliações, foram assinaladas e, posteriormente somadas para verificação da pontuação total de cada escala.

\section{Análise estatística}

A partir desses dados, os valores das escalas foram comparados através do índice de correlação de Pearson e a reta de regressão entre os resultados das avaliações.

\section{RESULTADOS}

Os resultados obtidos demonstraram uma correlação positiva excelente $(r: 0,86)$ entre o déficit de equilíbrio e o risco de quedas nos indivíduos avaliados (Figura 1). Esses resultados sugerem que quanto maior o desequilíbrio estático e dinâmico maior a propensão a quedas em atividades rotineiras. Esses indivíduos apresentavam uma capacidade reduzida de manter o equilíbrio em condições nas quais as informações sensoriais eram reduzidas.

\section{Discusão}

O cerebelo compara os comandos para o movimento transmitidos desde o córtex motor, com o desempenho motor real dos segmentos do corpo. Isto ocorre por meio de uma comparação entre a informação recebida desde o córtex e a informação obtida dos mecanismos de feedback periférico. Esse feedback propicia um contínuo input no que concerne à postura e ao equilíbrio, bem como a posição, velocidade, ritmo e força dos movimentos lentos dos segmentos corporais periféricos ${ }^{11,18}$.

Limites de estabilidade são definidos como o ângulo máximo a partir da vertical que pode ser tolerado sem perda de equilíbrio ${ }^{16}$.

Figura 1. Representação gráfica da reta de regressão da avaliação do equilibrio (BERG) e propensão à queda (EEEA). Índice de correlação de Pearson: r:0,86.

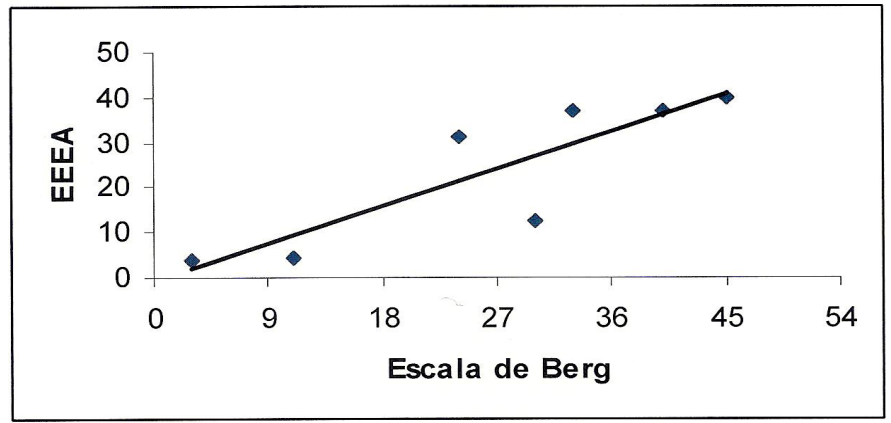


Portanto, o indivíduo é capaz de transferir o peso para frente e para trás ou de um lado para o outro sem perder o equilíbrio ou dar um passo ${ }^{19}$. Quanto mais largo o trajeto do reajuste postural, maior a instabilidade postural. Por exemplo, o paciente com ataxia costuma mostrar respostas hipermétricas, com trajetórias de balanço largas e pouca estabilidade postural ${ }^{20,21}$. Dessa forma a propensão ao risco de quedas nesses indivíduos será maior.

O desempenho de indivíduos normais quanto às informações sensoriais para o controle postural são variadas. Essa abordagem utiliza uma plataforma móvel, cercada por um ambiente visual também móvel ${ }^{22}$. Experimento semelhante foi conduzido para determinar o efeito das lesões cerebelares sobre a capacidade de reconsiderar as respostas posturais nas condições mutáveis de tarefa.

Crianças com encefalopatia crônica não evolutiva com sintomas cerebelares demonstraram uma capacidade significantemente reduzida de manter o equilíbrio em condições nas quais as informações sensoriais são reduzidas ${ }^{23}$. Isso apóia o conceito de que o cerebelo pode estar envolvido no processamento de informações associadas à organização central e a adaptação das respostas posturais.

Estudos que examinaram os fatores intrínsecos que provocam quedas incluiriam o exame da função do controle do equilíbrio, a fim de identificar pessoas propensas ao risco de quedas ${ }^{24,25}$. É importante considerar medidas com confiabilidade, validade e responsividade estabelecidas. Um dos testes que merecem atenção particular é a Escala de Equilíbrio de Berg. Este teste apresenta boa objetividade de testereteste, e consegue discriminar maior propensão a quedas. O declínio nas pontuações dessa escala foi associado a um risco elevado de queda, porém essa relação não é linear. $\mathrm{Na}$ amplitude de 56 a 54, cada ponto a menos na escala de Berg é associado a um aumento de 3 a $4 \%$ no risco de quedas. No entanto, na amplitude de 54 a 46, uma alteração de um ponto nessa escala foi associada a um aumento de 6 a $8 \%$ no risco de quedas. Abaixo de 36, o risco é próximo dos $100 \%$. Portanto, uma mudança de ponto na escala de Berg pode levar a uma previsão muito diferente de probabilidade de quedas, dependendo de onde a pontuação de linha-base se encontra na escala16. No presente estudo houve indivíduos com pontuação na escala de Berg de 5 a 35, o que significa que o risco de quedas se aproxima de $100 \%$.

Os resultados deste estudo evidenciaram uma diminuição do equilíbrio estático e dinâmico em to- dos os indivíduos avaliados pela escala de Berg. Esse desequilíbrio interfere diretamente nas repercussões funcionais desses indivíduos. Isso foi comprovado através da EEEA, uma vez que todos os indivíduos apresentaram baixas porcentagens em suas respostas, principalmente em atividades de vida diária.

Em estudo com idosos, a propensão de quedas nessa população, com relação causal de $12 \%$ de todos os óbitos na população geriátrica ${ }^{26}$. Mais de $70 \%$ das quedas ocorrem em casa, sendo que as pessoas que vivem só apresentam risco aumentado. Fatores ambientais podem ter papel importante em até metade de todas as quedas8.

Embora o desempenho dos pacientes fosse examinado em uma série limitada de condição ambiental, esses escores conseguiram representar o desempenho real em ambientes mais complexos. Dos sete pacientes participantes, quatro evidenciaram que caem numa freqüência média de 26 vezes ao ano, e os outros três participantes evidenciaram que caem quando ficam em pé, sem apoio.

\section{CONCLUSÃO}

Os dados do presente estudo evidenciaram que a marcha atáxica e déficits de coordenação proporcionam um desequilíbrio estático e dinâmico nesses indivíduos levando a maior propensão a quedas.

\section{REFERÊNCIAS BIBLIOGRÁFICAS}

1. Bower JM, Parsons LM. O Cerebelo - Reconsiderado. Sci Am Bras 2003;66-73.

2. Lundy-Ekman L. Neurociência: fundamentos para a reabilitação. 2 $2^{\text {a }}$ ed. Rio de Janeiro: Guanabara Koogan; 2004, 447p.

3. Lau KK, Au KM, Chen ML, Li HL, Sheng B, Chan AY. Spinocerebellar ataxia type 6. Hong Kong Med J 2005;11:207-9. 4. Montero LT. Avances en la comprensión de las ataxias hereditarias. Arq Neuropsiquiatr 1998; 61:175-88.

5. Margolis RL. The Spinocerebellar ataxias: order emerges from chaos. Curr Neurol Neurosci Rep 2002;2:447-56.

6. Van de Warrenburg BP, Steijns JA, Munneke M, Kremer BP, Bloem BR. Falls in degenerative cerebellar ataxias. Mov Disord 2005;20:497-500

7. Veiga Neto ER, Segura DCA. O cerebelo e as aferências da propriocepção inconsciente. Arq Cienc Saúde UNIPAR 2002;6:145-9.

8. Freitas EV, Py L, Cançado FAX, Gorzoni ML, Doll J. Tratado de Geriatria e Gerontologia. Rio de Janeiro: Guanabara Koogan, 2006, 1666p.

9. Brauer S, Burns Y, Galley P. Lateral reach: a clinical measure of medio-lateral postural stability. Physio Res Int 1999;4:81-8.

10. Duarte M, Zatsiorsky VM. Effects of body lean and visual information on the equilibrium maintenance during stance. Exp Brain Res 2002;146:60-9.

11. Shumway-Cook A, Woollacott MH. Controle Motor: Teoria e aplicações práticas. 2a ed. São Paulo: Manole, 2002, 592p. 
12. Kelly PJ, Stein J, Shafqat S, Eskey C, Doherty D, Chang Y, et al. Functional Recovery After Rehabilitation for Cerebellar Stroke. Stroke 2001;32:530-4.

13. Salmória JG, Marques LMPC, Chiquetti EMS. Exercícios de Frenkel adaptados e modificados no tratamento de paciente com marcha atáxica e incoordenacäo motora: relato de caso. Arq Cienc Saúde UNIPAR 2002;6:151-7.

14. Latash, M, Ferreira SMS, Wieczorek SA, Duarte M. Movement sway: changes in postural sway during a voluntary shifts of the center of pressure. Exp Brain Res 2003;150:314-24.

15. Fuller GF. Problem-oriented diagnosis: falls in the elderly. Am Fam Phys 2000;61:2159-68.

16. Miyamoto ST, Lombardi Jr I, Berg KO, Ramos LR, Natour J. Brazilian version of the Berg balance scale. Braz J Med Biol Res 2004;37:1411-21.

17. Powell LE, Myers AM. The activities-specific balance confidence (ABC) scale. J Gerontolol 1995;50:28-35.

18. Umphred DA. Fisioterapia Neurológica. $2^{\underline{a}}$ ed. São Paulo: Manole, 2004, 1118p.

19. Polastri PF, Godoi D. Controle postural em crianças: oscilação corporal e freqüência de oscilação. Rev Paul Ed Fís 2000;14:55-64.
20. Harris-Love MO, Siegel KL, Paul SM, Benson K Rehabilitation management of Friedreich ataxia: lower extremity forcecontrol variability and gait performance. Neurorehabil Neural Repair 2004;18:117-24

21. Morales AO, Vilatela AME. Ataxia: efectos psicosociales. Arch Neurocienc 2001;6:108-11.

22. Perennou D, Decavel P, Manckoundia P, Penven Y, Mourey F, Launay F, et al. Evaluation of balance in neurologic and geriatric disorders. Ann Readapt Med Phys 2005;48:317-35.

23. Nashner LM, Shumway-Cook A, Marin O. Stance posture control in select groups of children with cerebral palsy: déficits in sendory organization and muscular coordination. Exp Brain Res 1983;49:393-409.

24. Krishchiunas AI, Savitskas RIU. Falls in early in-patient rehabilitation of stroke patients. Zh Nevrol Psikhiatr Im S S Korsakova 2004;Suppl 11:47-50.

25. Huang M, Burgess R, Weber M, Greenwald N. Performance of balance impaired elders on three balance tests under two visual conditions. J Geriatr Phys Ther 2006;29:5-9.

26. Baraff LJ, Schriger DL, Bass JW, Fleisher GR, Klein JO, McCracken GH Jr., et al. Practice guideline for the ED Management of falls in community - dwelling elderly persons. Ann Emerg Med 1997;30:480-92. 